\title{
Diffusion Time-Scale of Porous Pressure-Sensitive Paint
}

\author{
AIAA Journal, Vol. 39, No. 12, pp. 2400-2402 \\ Tianshu $\operatorname{Liu}^{\dagger}$ \\ NASA Langley Research Center, Hampton, VA 23681-2199 \\ t.liu@larc.nasa.gov, Tel: 757-864-4639,Fax: 757-864-4799 \\ Norikazu Teduka $^{*}$ and Masaharu Kameda ${ }^{* *}$ \\ Tokyo University of Agriculture and Technology, Koganei, Tokyo 184-8588, Japan \\ Keisuke Asai ${ }^{\ddagger}$ \\ National Aerospace Laboratory, Chofu, Tokyo 182-8522, Japan.
}

\footnotetext{
${ }^{\dagger}$ Research Scientist, Model Systems Branch, MS 238, Member AIAA

* Graduate Student, Department of Mechanical Systems Engineering

** Associate Professor, Department of Mechanical Systems Engineering

$\$$ Group Leader, Advanced Measurement Technology Group, Associate Fellow AIAA
} 


\section{Introduction}

Pressure-sensitive paint (PSP) is an optical pressure sensor that utilizes the oxygen quenching of luminescence. PSP measurements in unsteady aerodynamic flows require fast time response of the paint $[1,2,3]$. There are two characteristic time-scales that are related to the time response of PSP. One is the luminescent lifetime representing an intrinsic physical limit for the achievable temporal resolution of PSP. Another is the time-scale of oxygen diffusion across the PSP layer. When the time-scale of oxygen diffusion is much larger than the luminescent lifetime, the time response of PSP is controlled by oxygen diffusion. In a thin homogenous polymer layer where diffusion is Fickian, the oxygen concentration $\left[\mathrm{O}_{2}\right]$ can be described by the diffusion equation in one-dimension,

$$
\frac{\partial\left[O_{2}\right]}{\partial t}=D_{m} \frac{\partial^{2}\left[O_{2}\right]}{\partial z^{2}}
$$

where $D_{m}$ is the diffusivity of oxygen mass transfer in the polymer, $t$ is the time, and $z$ is the coordinate normal to the layer. For an arbitrary time-dependent change of the oxygen concentration at the air-paint interface, the complete convolution-type solution of Eq. (1) with the suitable boundary conditions can be obtained by using the Laplace transform $[4,5]$. The special trigonometrical-series-type solutions for a step change and a sinusoidal change of oxygen were used for PSP dynamic analysis [1,2,3]. The solution of the diffusion equation gives a wellknown square-law estimate for the diffusion time-scale $\tau_{\text {diff }}$ through a PSP layer,

$$
\tau_{\text {diff }} \propto h^{2} / D_{m}
$$

where $h$ is the PSP layer thickness. The response time of a homogenous PSP is proportional to the square of the layer thickness and inversely proportional to the diffusivity of mass transfer $D_{m}$. This estimate is correct for a conventional homogenous PSP. 
Compared with a conventional PSP, a porous PSP has a much shorter diffusion response time ranging from $18 \mu \mathrm{s}$ to $500 \mu \mathrm{s}$ [6]. The shorter diffusion time-scale is essentially related to an enlarged air-polymer interface in a porous PSP. Interestingly, recent measurements of the response time for three polymers GP197, GP197/BaSO4 mixture and Poly(TMSP) showed that the classical square-law estimate (2) does not hold for porous PSPs [7, 8]. As shown in Fig. 1, measurements gave the power-law relations $\tau_{\text {diff }} \propto h^{1.83}$ for GP197, $\tau_{\text {diff }} \propto h^{1.07}$ for GP197/BaSO4 mixture, and $\tau_{\text {diff }} \propto h^{0.29}$ for Poly(TMSP) at a temperature of $313.1 \mathrm{~K}$. For the GP197 silicone polymer, the power-law exponent is close to 2 as predicted by the classical estimate. However, the power-law exponents for the porous polymer materials GP197/BaSO4 mixture and Poly(TMSP) are significantly smaller than 2. In addition, Fig. 2 shows that the power-law exponent for the polymer Poly(TMSP) linearly increases with temperature over a temperature range from $293.1 \mathrm{~K}$ to $323.1 \mathrm{~K}$. Unfortunately, a comprehensive and universal theory of diffusion in porous materials has not been developed yet. In order to understand the time response of a porous PSP, nevertheless, this note attempts to derive the expressions for the effective diffusivity and the diffusion response time of a porous polymer layer from a standpoint of phenomenology.

\section{Effective Diffusivity}

Diffusion in a porous material can be considered as a diffusion problem in a two-phase system made up of one disperse phase and one continuous polymer. In PSP, the disperse phase is composed of numerous pores filled with air. An element of a porous polymer layer of the length $l$, width $l$, and thickness $h$ is considered, as shown in Fig. 3. The coordinate $z$ is normally directed to the polymer layer from the upper surface of the layer. We assume that many 
cylindrical (tube-like) pores are distributed in the element and the pores are oriented in the $z$ direction. The effective radius and depth of a pore are denoted by $r_{\text {pore }}$ and $h_{\text {pore }}$, respectively. The radius of a pore is much larger than the size of a molecule of oxygen. In general, the depth of a pore is smaller than the layer thickness, i.e., $h_{\text {pore }} \leq h$. For simplicity of expression, the normal directional derivative of the oxygen concentration $\left[\mathrm{O}_{2}\right]$ at the air-polymer interface is denoted by

$$
v_{n}(z)=\frac{\partial\left[\mathrm{O}_{2}\right]}{\partial n}
$$

The effective diffusivity $D_{m e f f}$ of the porous polymer layer with cylindrical pores is given by a balance equation between the mass transfer through the apparent homogenous upper surface and the total mass transfer across the air-polymer interface,

$$
\begin{gathered}
D_{\text {meff }} l^{2} v_{n}(0)=D_{m}\left(l^{2}-N_{\text {pore }} \pi r_{\text {pore }}^{2}\right) v_{n}(0)+D_{m} N_{\text {pore }} \pi r_{\text {pore }}^{2} v_{n}\left(h_{\text {pore }}\right) \\
+D_{m} N_{\text {pore }} 2 \pi r_{\text {pore }} \int_{0}^{h_{\text {pore }}} v_{n}(z) d z
\end{gathered},
$$

where $N_{\text {pore }}$ is the total number of the pores in the element and $D_{m}$ is the diffusivity of the polymer continuum. The integral term in Eq. (4) is the total mass transfer across the peripheral surface of the pores in the element. Thus, the effective diffusivity $D_{\text {meff }}$ is given by

$$
\begin{aligned}
D_{\text {meff }} / D_{m}=1 & +\left[v_{n}\left(h_{\text {pore }}\right) / v_{n}(0)-1\right] N_{\text {pore }} \pi r_{\text {pore }}^{2} l^{-2} \\
& +N_{\text {pore }} 2 \pi r_{\text {pore }} l^{-2} v_{n}^{-1}(0) \int_{0}^{h_{\text {pore }}} v_{n}(z) d z
\end{aligned} .
$$

In a simplified case where $v_{n}(z)=$ cons tant across the thin layer, Eq. (5) becomes

$$
D_{m e f f} / D_{m}=1+2 a_{V} r_{\text {pore }}^{-1} h
$$


where $a_{V}=N_{\text {pore }} \pi r_{\text {pore }}^{2} h_{\text {pore }} l^{-2} h^{-1}$ is the volume fraction of the cylindrical pores in the polymer layer. Eq. (6) indicates an increase of the effective diffusivity that is proportional to the volume fraction of the pores and a ratio between the polymer layer thickness and the radius of the pore. The expression (6) for $D_{\text {meff }}$ is valid only for an ideal porous polymer layer with the straight cylindrical pores oriented normally. Nevertheless, this model should be generalized for real porous polymers where topology of the pores is often highly complicated.

In a more realistic model, the topological structure of a pore is considered as a highly convoluted and folded tube in the polymer layer while the cross-section of the tube remains unchanged. The integral in Eq. (5) should be replaced by the integral along the path of the highly convoluted tube-like pore. In this case, the concept of the fractal dimension should be introduced because the length of the highly convoluted tube is no longer proportional to the linear length scale of the tube in the $z$-direction (e.g. $h_{\text {pore }}$ ) [9]. According to the length-area relation for a fractal path, the integral along the path is proportional to $A_{\text {pore }}^{d_{f r} / 2}$ or $h_{\text {pore }}^{d_{f r}}$, where $d_{f r}\left(1 \leq d_{f r}<2\right)$ is the fractal dimension of the path of the pore and $A_{\text {pore }} \propto h_{\text {pore }}^{2}$ is the characteristic area covering over the path. Loosely speaking, the fractal dimension represents the degree of complexity of the pore pathway. In order to take the fractal nature of the pores into account, Eq. (5) is generalized by using a Riemann-Liouville fractional integral of the order $d_{f r}$, i.e., $[10]$

$$
\begin{aligned}
D_{\text {meff }} / D_{m}=1 & +\left[v_{n}\left(h_{\text {pore }}\right) / v_{n}(0)-1\right] N_{\text {pore }} \pi r_{\text {pore }}^{2} l^{-2} \\
& +N_{\text {pore }} 2 \pi r_{\text {pore }} l^{-2} v_{n}^{-1}(0) \int_{0}^{h_{\text {pore }}} v_{n}(z)(d z)^{d_{f r}} .
\end{aligned}
$$


Note that a unitary constant with the dimension $\left[m^{1-d_{f r}}\right]$ is implicitly embedded in the third term in the right-hand side of Eq. (7) to make Eq. (7) dimensionally consistent. This dimensional constant is implicitly contained in all the results derived from Eq. (7). In a simplified case where $v_{n}(z)=$ constant across the thin layer, a generalized expression for $D_{\text {meff }}$ is

$$
\frac{D_{m e f f}}{D_{m}}=1+\left.\frac{2 a_{V} r_{\text {pore }}^{-1}}{\Gamma\left(1+d_{f r}\right)} \frac{h_{\text {pore }}}{h}\right|^{d_{f r}-1} h^{d_{f r}}
$$

where $\Gamma\left(1+d_{f r}\right)$ is the gamma function. Here, $h_{p o r e}$ is interpreted as a linear length scale of the convoluted tube in the $z$-direction and $a_{V}$ is the volume fraction of the apparent cylindrical pores. The expression (8) clearly shows that the effective diffusivity $D_{\text {meff }}$ is not only proportional to $h^{d_{f r}}$, but also related to the parameters of porosity $a_{V} r_{p o r e}^{-1}$ and $h_{\text {pore }} / h$. For $d_{f r}=1$, Eq. (8) is simply reduced to Eq. (6).

\section{Diffusion Time-Scale}

For a porous polymer layer where diffusion is Fickian under some microscopic assumptions $[11,12]$, the diffusion equation (1) is still a valid phenomenological model as long as the diffusivity $D_{m}$ is replaced by the effective diffusivity $D_{m e f f}$. Hence, the estimate for the diffusion time-scale for a porous PSP layer is

$$
\tau_{d i f f} \propto \frac{h^{2} / D_{m}}{1+\left.\frac{2 a_{V} r_{p o r e}^{-1}}{\Gamma\left(1+d_{f r}\right)} \frac{h_{\text {pore }}}{h}\right|^{d_{f r}-1} h^{d_{f r}}} .
$$


Eq. (9), a generalized form of Eq. (2), clearly illustrates how the parameters of porosity $a_{V} r_{\text {pore }}^{-1}$ and $h_{p o r e} / h$, and the fractal dimension $d_{f r}$ affect the response time of a porous PSP. For $a_{V} r_{\text {pore }}^{-1}<<1$ or $h_{\text {pore }} / h<<1$, Eq. (9) naturally approaches to the classical square-law estimate (2) for a homogenous polymer layer. On the other hand, for $a_{V} r_{\text {pore }}^{-1}>1$ and $h_{\text {pore }} / h \approx 1$, another asymptotic estimate for $\tau_{\text {diff }}$ is a simple power-law

$$
\tau_{d i f f} \propto h^{2-d_{f r}} / D_{m}
$$

The estimate $(10)$ is asymptotically valid for a very porous polymer layer. The exponent in the power-law relation between the response time $\tau_{\text {diff }}$ and thickness $h$ deviates from 2 by the fractal dimension $d_{f r}$ because of the presence of the fractal pores in the polymer layer. The relation (10) provides an explanation for the experimental finding that the exponent $q$ in the power-law relation $\tau_{\text {diff }} \propto h^{q}$ is less than 2 for porous PSPs [7,8]. Also, this relation can serve as a useful tool to extract the fractal dimension of the tube-like pores in a very porous polymer layer from measurements of the diffusion response time. The fractal dimension $d_{f r}$ of the pore in the polymer Poly(TMSP) is $d_{f r}=1.71$. For GP197/BaSO4 mixture, the fractal dimension $d_{f r}$ is close to one. In addition, based on the experimental results shown in Fig. 2, one knows that the fractal dimension $d_{f r}$ for the polymer Poly(TMSP) linearly decreases with temperature in a temperature range from $293.1 \mathrm{~K}$ to $323.1 \mathrm{~K}$. This implies that the geometric structure of the pore in Poly(TMSP) may be altered by temperature change. Note that the diffusivity $D_{m}$ of oxygen mass transfer is also temperature-dependent, but it is independent of the coating thickness $h$. Therefore, the experimental results in Fig. 2 mainly reflect the effect of temperature on the geometric structure of the pores in the polymer rather than the diffusivity. 


\section{Conclusions}

The Note develops a simple phenomenological model for the effective diffusivity of a porous PSP and gives a new expression that clearly illustrates the relationship between the diffusion time-scale and the fractal dimension of the pores. The theoretical results can not only explain why a porous PSP is able to achieve a very fast time response, but also quantitatively show how the fractal dimension and the parameters of porosity affect the response time. For very porous PSPs, the classical square-law estimate of the diffusion time-scale should be replaced by the generalized relation derived in the Note.

\section{References}

[1] Carroll, B. F., Abbitt, J. D., Lukas, E. W. and Morris, M. J., "Step Response of Pressure Sensitive Paints," AIAA J, Vol. 34, No. 3, 1996, pp.521-526.

[2] Winslow, N. A., Carroll, B. F., and Kurdila, A. J., "Model Development and Analysis of the Dynamics of Pressure-Sensitive Paints," AIAA J., Vol. 39, No. 4, 2001, pp. 660-666.

[3] Fonov, S., Mosharove, V., Radchenko, V., Engler, R. and Klein, C., "Application of the PSP for Investigation of the Oscillating Pressure Fields," AIAA Paper 98-2503, June, 1998, Albuquerque, NM.

[4] Carslaw, H. S. and Jaeger, J. C., "Conduction of Heat in Solids (Second Edition)," Oxford Science Publications, Oxford, 2000, Chapter XII.

[5] Crank, J., "The Mathematics of Diffusion (Second Edition)," Oxford Science Publications, Oxford, 1995, Chapter 2. 
[6] Sakaue, H., Gregory, J. W., Sullivan, J. P. and Raghu, S., "Porous Pressure Sensitive Paint for Characterizing Unsteady Flow Fields," AIAA J., 2001, (submitted).

[7] Teduka, N., "Fast Response Pressure Sensitive Coatings for Unsteady Measurements," The Master Thesis, Department of Mechanical Systems Engineering, Tokyo University of Agriculture and Technology, 2001.

[8] Asai, K., Nakakita, K., Kameda, M. and Teduka, N., "Recent Topics in Fast-Responding Pressure-Sensitive Paint Technology at National Aerospace Laboratory," The 19th International Congress on Instrumentation in Aerospace Simulation Facilities (ICIASF), Aug. 27-30, 2001, Cleveland, $\mathrm{OH}$.

[9] Mandelbrot, B. B., “The Fractal Geometry of Nature,” W. H. Freeman, New York, 1982.

[10] Nishimoto, K., "Fractional Calculus," Descartes Press Co., Koriyama, Japan, 1991, Chapter 1.

[11] Cunningham, R. E. and Williams, R. J. J., "Diffusion in Gases and Porous Media," Plenum Press, New York, 1980, Chapter 1.

[12] Neogi, P, "Transport Phenomena in Polymer Membranes," in Diffusion in Polymers, ed. P. Neogi, Marcel Dekker, Inc., New York, 1996, Chapter 4. 


\section{Captions}

Figure 1. Power-law relationship between response time and coating thickness for three polymers GP197, GP197/BaSO4 mixture and Poly(TMSP) at a temperature of $313.1 \mathrm{~K}$.

Figure 2. The exponent of the power-law relation between time-scale and coating thickness for the polymer Poly(TMSP) as a function of temperature.

Figure 3. An element of a porous polymer layer. 


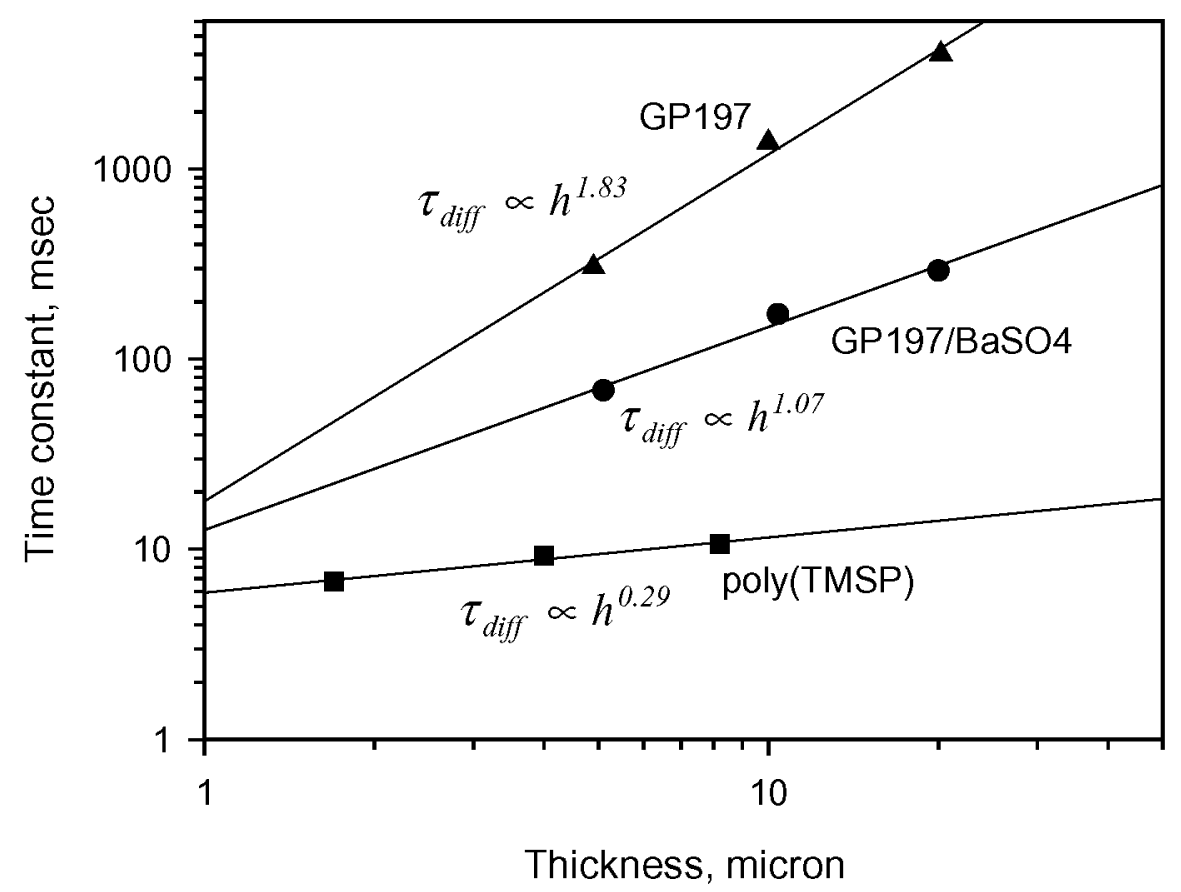

Figure 1. Power-law relationship between response time and coating thickness for three polymers GP197, GP197/BaSO4 mixture and Poly(TMSP) at a temperature of 313.1K. 


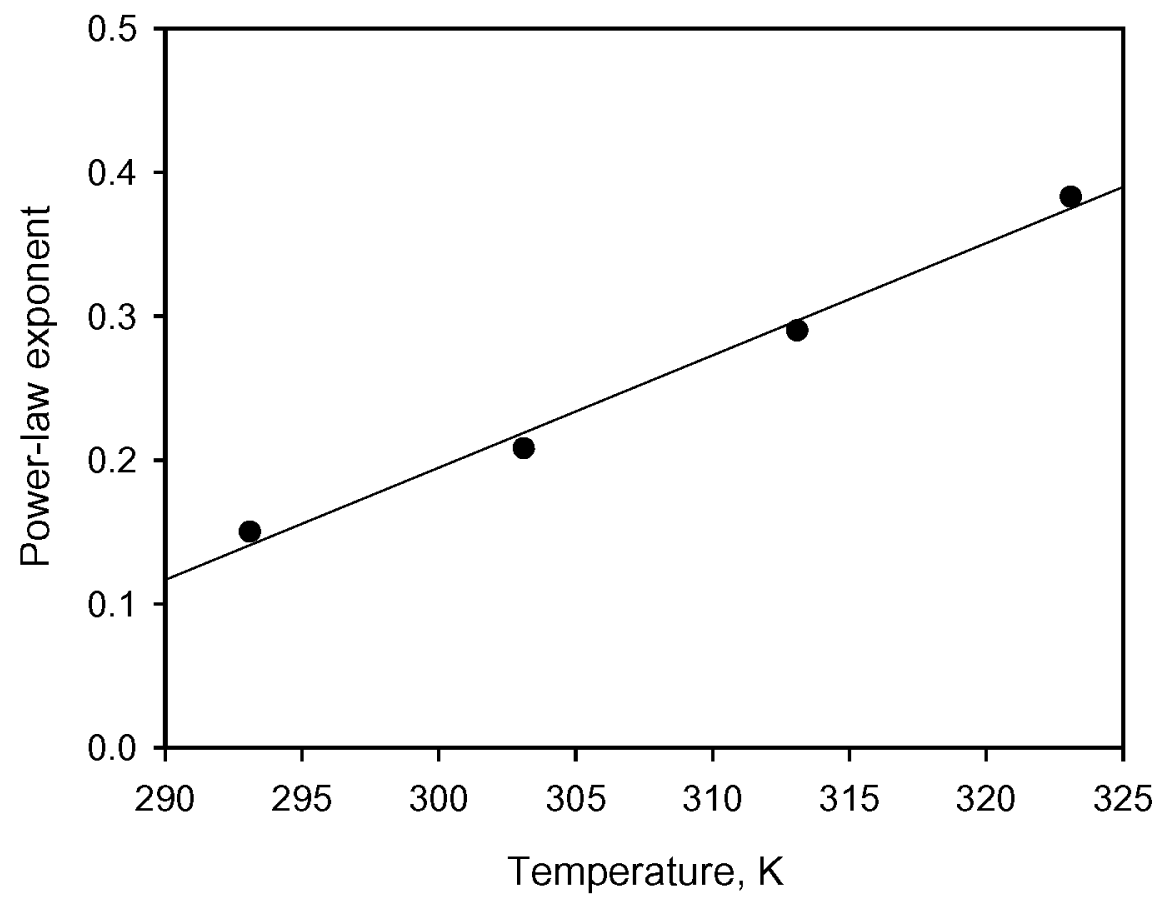

Figure 2. The exponent of the power-law relation between time-scale and coating thickness for the polymer Poly(TMSP) as a function of temperature. 


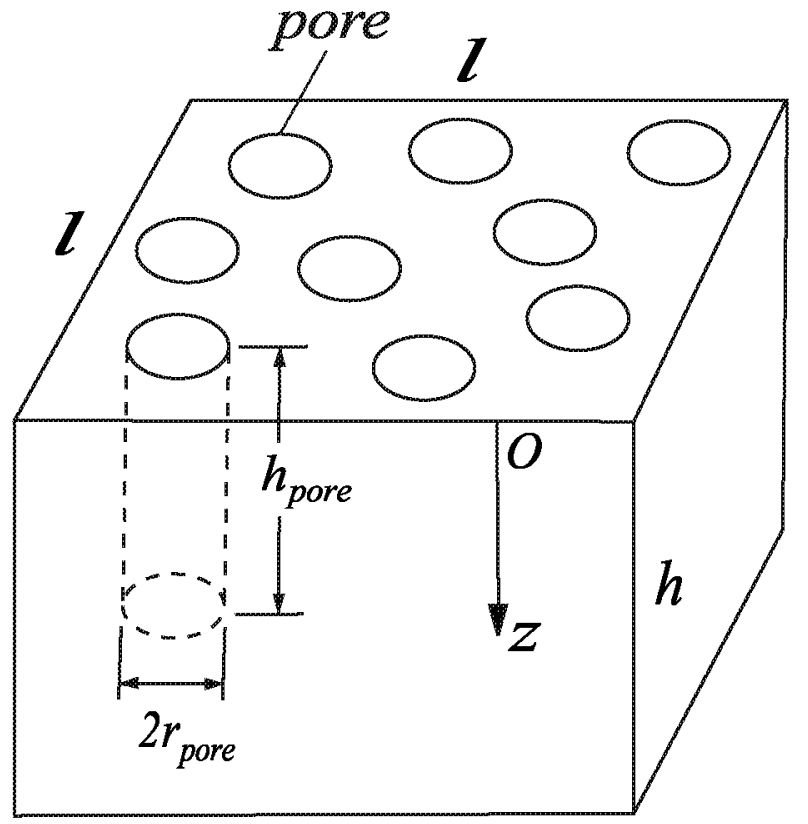

Figure 3. An element of a porous polymer layer. 